\title{
High Performance Liquid Chromatographic Determination of Alcohol in Alcoholic Drinks with a Conductometric Detector
}

\author{
Tadashi Iwachido*, Kenji IshIMARU** and Kyoji TôEI** \\ * College of Liberal Arts, Okayama University, Tsushima, Okayama 700 \\ ** Department of Chemistry, Faculty of Science, Okayama University, \\ Tsushima, Okayama 700
}

Keywords Alcohol, alcoholic drinks, conductometric detection, high performance liquid chromatography

In high performance liquid chromatography, conductometric detectors have been used only for the detection of electrolytes in solution. Non-electrolytes or neutral compounds are detected by other instruments, such as refractive index detectors, UV-VIS absorption detectors, or electrochemical detectors. ${ }^{1}$ Non-electrolytes, however, are detectable as reversed peaks when electrolyte solutions are used as the mobile phase and conductometers are used as the detectors. This letter describes how the amount of ethanol in alcoholic drinks can be successfully determined by means of reversed phase chromatography by utilizing a conductometer as the detector.

\section{Experimental}

The apparatus used for this work was a LC-5A solvent delivery system (Shimadzu Corp.) equipped with a conductometric detector (Conductomonitor 701, Laboratory Data Control) and a Rheodyne 7125 syringe-loading injector (Rheodyne Inc.) with a sampleloop of an effective volume of $5 \mu \mathrm{l}$. Aqueous alcohol solutions were chromatographed on an octadecylsilanemodified silica gel column $(5 \mu \mathrm{m}, 150 \times 4.6 \mathrm{~mm}$ I.D., Gasukuro Kogyo Inc.) by using an aqueous sodium chloride solution $\left(10^{-2} \mathrm{~mol} \mathrm{dm}^{-3}\right)$ as the mobile phase at a flow rate of $1.0 \mathrm{~cm}^{3} \mathrm{~min}^{-1}$ (pressure drop: about $100 \mathrm{~kg} \mathrm{~cm}^{-2}$ ). The chromatograms were recorded on a 3390A Reporting Integrator (HewlettPackard). The alcohol solutions were pre-treated with a disposable alumina B cartridge (Sep-Pak, Waters Associates). All the measurements were carried out at room temperature.

\section{Results and Discussion}

Typical chromatograms obtained with aqueous alcohol solutions were depicted in Fig. 1. The chromatogram (A) was obtained with a mixture of ethanol and water (ethanol/water $20 / 40 \mathrm{v} / \mathrm{v}$ ). Water and ethanol were observed as reversed peaks separately at the retention times of 1.6 and $3.4 \mathrm{~min}$, respectively.

In Fig. 2, the peak areas of ethanol and water are plotted against the ethanol content $(\% \mathrm{v} / \mathrm{v})$. The peak area of ethanol (open circle) increased linearly but that of water (closed circle) decreased linearly with an increase in the ethanol concentration. The retention time of ethanol slightly decreased with an increase in the ethanol concentration.

The use of concentrated electrolyte solutions as the mobile phase markedly enhanced the sensitivity for

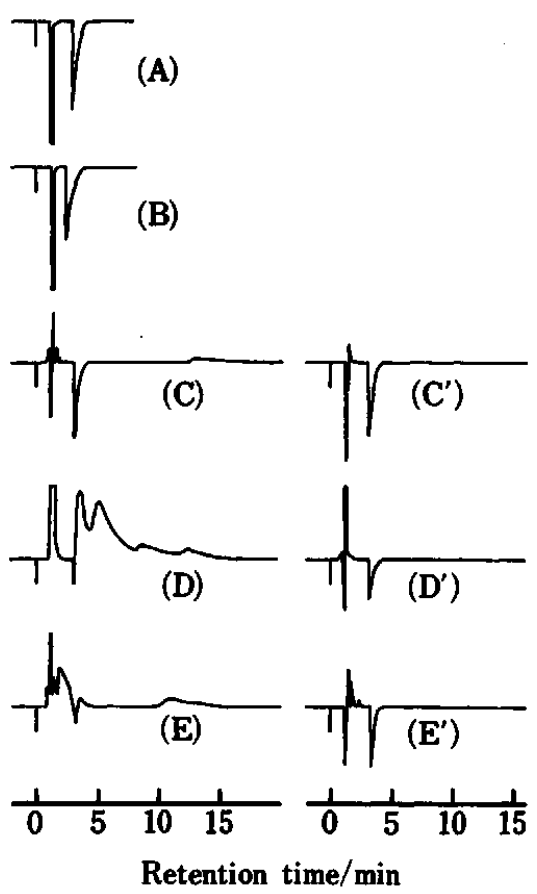

Fig. 1 High performance liquid chromatograms of alcohol drinks. (A) aqueous ethanol solution $20 \%$ (v/v); (B) whisky; (C) sake; (D) wine; (E) beer. ( $\left.C^{\prime}\right),\left(D^{\prime}\right)$ and $\left(E^{\prime}\right)$ : drinks pretreated with a disposable alumina column. 


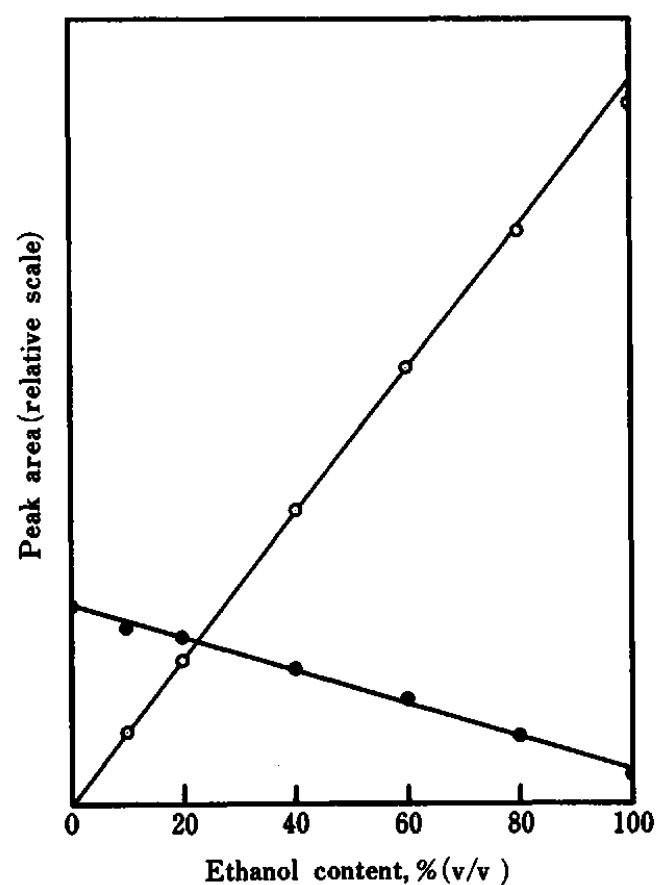

Fig. 2 Plots of the peak areas of ethanol and water vs. ethanol contents. Open circle, ethanol; closed circle, water.

ethanol, but resulted in noisy chromatograms. Therefore, $\boldsymbol{x}$ mobile phase of an appropriate electrolyte concentration must be chosen so as to be suited for the purpose. The species of the electrolyte showed no influence on the peak area when their equivalent ionic conductivities were taken into account.

Chromatograms of several alcoholic drinks are shown in Fig. 1 (B-E). The chromatogram of whisky (B) was quite simple and no peaks other than ethanol and water were detected. The chromatograms of sake (C), a Japanese fermented liquor made from rice, wine (D), and beer (E) exhibited complex patterns. The normal peaks were assigned to electrolytes. The chromatographic separation of ethanol from these electrolytes was almost satisfactory, except in the case of beer. The chromatograms, C, D, and $E$, show that these drinks contain many kinds of brewing substances which are eluted later than ethanol. The presence of these substances is not favorable for the rapid determination of ethanol. The chromatograms, $C^{\prime}, D^{\prime}$, and $E^{\prime}$, indicate that these substances are removed by pre-treating these drinks with a short alumina column. Ethanol was not lost on this pre-treatment.

The effects of the inorganic and organic concomitant substances $^{2}$ on the determination of ethanol were investigated. Table 1 shows that the presence of these substances do not interfere with the determination.
Table 1 Effect of concomitant substances on the determination of ethanol

\begin{tabular}{llc}
\hline $\begin{array}{c}\text { Concomitant } \\
\text { substance }\end{array}$ & $\begin{array}{c}\text { Added } / \\
\text { mol dm }\end{array}$ & $\begin{array}{c}\text { Relative peak } \\
\text { area, \% }\end{array}$ \\
\hline None $^{\mathrm{a}}$ & 0.1 & 100.0 \\
$\mathrm{KCl}$ & 0.05 & 100.4 \\
$\mathrm{~K}_{2} \mathrm{SO}_{4}$ & 0.033 & 100.4 \\
$\mathrm{~K}_{3} \mathrm{PO}_{4}$ & 0.05 & 100.4 \\
Sodium acetate & 0.005 & 100.4 \\
Tartaric acid & 0.05 & 100.0 \\
$n$-Butyric acid & 1.0 & 99.6 \\
Dextrose & 1.0 & 99.8 \\
Fructose & 0.1 & 99.8 \\
Ethyl formate & 0.5 & 99.8 \\
Ethyl acetate & 99.6 \\
\hline
\end{tabular}

a. Ethanol solution: $20 \%(v / v)$.

Table 2 The concentration of ethanol in alcoholic drinks

\begin{tabular}{lccc}
\hline \multirow{2}{*}{ Drinks } & \multicolumn{3}{c}{ Ethanol content, \% (v/v) } \\
\cline { 2 - 4 } & $\begin{array}{c}\text { Certified } \\
\text { value }\end{array}$ & $\begin{array}{c}\text { Proposed } \\
\text { method }\end{array}$ & $\begin{array}{c}\text { Oxidation } \\
\text { method }\end{array}$ \\
\hline Whisky & 37 & 36.9 & 36.6 \\
Sake & $15-16$ & 14.9 & 14.5 \\
Wine & $<14$ & 12.8 & 12.2 \\
Beer & 4.5 & 4.4 & 4.5 \\
\hline
\end{tabular}

Ethanol contents in four kinds of drinks are shown in Table 2. The values were in good agreement with those obtained by means of an oxidation method, in which the drinks are distilled and the distillates are titrated with potassium dichromate solution by utilizing barium 4-diphenylaminesulfonate as the indicator. ${ }^{3}$ The ethanol contents determined by the proposed method also agreed well with those printed on the labels. The chromatographic response was linear over the ethanol concentration range $2-100 \%(v / v)$. The relative standard deviation for 11 runs at $20 \%(\mathrm{v} / \mathrm{v})$ ethanol solution was $0.51 \%$. These findings indicate that the proposed method is successfully applicable to the determination of ethanol in alcoholic drinks.

\section{References}

1. T. M. Vickrey (ed.), "Liquid Chromatography Detectors", Marcel Dekker, New York (1983).

2. "Jozo Seibun Ichiran", p. 279, Nihon Jozo Kyokai (1977).

3. "Eisei Shikenho", p. 284, ed. Nihon Yakugakukai, Kanehara Shuppan, Tokyo (1977). 